cocktail to which the organisms were sensitive suggests that Pa was cleared earlier and more effectively in the phage-treated animals; this was confirmed by significant differences in bacterial load at the earlier, 24 hour time point. Further work is underway to explore the therapeutic potential of bacteriophage in pulmonary Pa infection.

\section{S104 EXPOSURE TO WELDING FUME AND ADHESION OF STREPTOCOCCUS PNEUMONIAE TO A549 ALVEOLAR CELLS}

doi:10.1136/thoraxjnl-2012-202678.109

${ }^{1} \mathrm{R}$ Suri, ${ }^{2} \mathrm{~K}$ Palmer, ${ }^{3} \mathrm{JAS}$ Ross, ${ }^{2} \mathrm{D}$ Coggon, ${ }^{1} \mathrm{~J}$ Grigg. ${ }^{1}$ Blizard Institute of Cell and Molecular Sciences, London, UK; ${ }^{2}$ MRC Environmental Epidemiology Unit, Community Clinical Sciences, Southampton, UK; ${ }^{3}$ Department of Environmental and Occupational Medicine, University of Aberdeen, Aberdeen, UK

Background and Objectives Epidemiological studies suggest that that occupational exposure to welding fumes (WF), increases susceptibility to invasive pneumococcal disease. ${ }^{1}$ The alveoli are a site for invasive disease caused by Streptococcus pneumoniae (Sp). To date the mechanism whereby welding fumes increases susceptibility to pneumococcal pneumonia is unknown. Platelet activating factor receptor (PAFR) is a host entry receptor for Sp in airway cells exposed to particulate matter air pollution. ${ }^{2}$ We assessed the hypothesis that welding fumes increase adhesion of Sp to airway cells via PAFR.

Methods Fume from a mild steel hyperbaric welding operation (WF) were collected from filtres and resuspended in PBS. Monolayers of the alveolar epithelial cell line, A549, were exposed to either WF alone or WF + a PAFR blocker (CV3988) for $3 \mathrm{~h}$. Cells were then exposed to $\mathrm{Sp}$ for $2 \mathrm{~h}$. Adherent and internalised bacteria were assessed by quantitative culture. Data were expressed as a mean \pm SEM of $>4$ replicates and were compared by $t$ test.

Results WF increased pneumococcal adhesion to A549 cells (Fig $1 \mathrm{~A})$ in a dose-dependent manner $(* * p<0.01$ vs. control). Blocking PAFR with CV3988 attenuated WF-stimulated adhesion $(* * p<0.005$, Fig 1B).

Conclusion Increased PAFR-medicated pneumococcaladhesion to lower airway cells is a putative mechanism for the association between exposure to WF and vulnerability to pneumococcal pneumonia.

1. Palmer, KT; Poole, J; Ayres, J G; Mann, J; Burge, P S; Coggon, D. Exposure to Metal Fume and Infectious Pneumonia. American Journal of Epidemiology 157, 227-233 (2003).

2. Mushtaq, $\mathrm{N}$ et al. Adhesion ofStreptococcus pneumoniae to human airway epithelial cells exposed to urbanparticulate matter. The Journal of allergy and clinical immunology 127,1236-42 (2011)
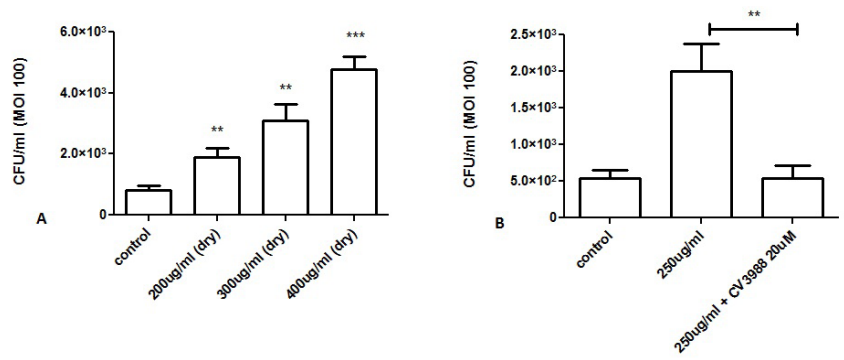

Abstract S104 Figure 1

\section{S105 ENDOTOXIN SPECIFIC IGG2 ANTIBODIES IMPAIR BACTERIAL KILLING IN NON CYSTIC FIBROSIS BRONCHIECTASIS PATIENTS COLONISED WITH PSEUDOMONAS AERUGINOSA}

doi:10.1136/thoraxjnl-2012-202678.110

'D Whitters, ${ }^{2} \mathrm{TJ}$ Wells, ${ }^{2} \mathrm{IR}$ Henderson, ${ }^{1}$ RA Stockley. ${ }^{1}$ University Hospitals Birmingham NHS Trust, Birmingham, UK; ${ }^{2}$ School of Immunity and Infection, University of Birmingham, Birmingham, UK
Introduction We have previously identified patients colonised with Pseudomonas aeruginosa (PA), in whom strains isolated from their sputum cannot be killed by their serum, yet are fully sensitive to healthy control serum (HCS). Addition of inhibitory patient serum to HCS also impaired killing of PA.

Fractionation of patient serum identified that $\operatorname{IgG}$ was responsible for the inhibition. FACS demonstrated excessive binding of IgG2 from patient serum to autologous strains.

Visualisation of lipopolysaccharide (LPS) isolated from bacterial strains by silver staining, showed that strains from patients with inhibitory serum demonstrated detectable $O$ antigen expression - a component of the LPS cell wall of PA.

We aimed to confirm this relationship between inhibitory IgG2 and LPS expression by PA.

Methods Anti-LPS antibodies were removed from inhibitory serum (S4) by binding LPS from PA isolated from patient sputum, to polymyxin-B agarose overnight. Inhibitory serum was then passed over the LPS bound column. Antibodies specific for LPS bound to the column, and the flow through fractions of serum were collected. Bound antibody was subsequently buffer exchanged into PBS.

LPS was isolated from a PA strain resistant to patient sera (B4) and attached to a 96 well plate. ELISA was performed by adding dilutions of patient or HCS to the plate followed by anti-human IgG2 conjugated to alkaline phosphotase. Results were derived 30 minutes after addition of developer.

Results LPS removal of IgG from the patient's serum restored bacterial killing. Adding the eluted antibody to HCS impaired killing. This confirmed that inhibitory IgG2 is LPS specific. The patients' serum that blocked bacterial killing had high titres of IgG2 to LPS compared to those from patients with bactericidal serum (fig. 1)

Conclusion We have established that PA strains deficient in LPS O antigen are generally sensitive to killing by patients' serum, whilst the presence of $O$ antigen leads to serum resistance mediated by IgG2

Results indicate that PA LPS $O$ antigen repeats are central to serum resistance of autologous strains but not normal HCS. Current data indicates that bacterial killing is impaired in subjects colonised with PA expressing $O$ antigen due to overproduction of LPS specific IgG2.

\section{S106 INFLUENZA INFECTION OF HUMAN LUNG MACROPHAGES INCREASES PDL1 EXPRESSION}

doi:10.1136/thoraxjnl-2012-202678.111

KJ Staples, CM Spalluto, RT McKendry, TMA Wilkinson. University of Southampton Faculty of Medicine, Southampton, United Kingdom

Background \& Objective Influenza infection has recently been shown to cause rapid functional impairment of CD8 $+\mathrm{T}$ cell responses in a murine infection model via the PD1/PDL1 pathway. ${ }^{1}$ In this mouse model, it was the induction of PDL1 that was required for this impairment of CD8+ function. A previous study suggested that the anti-inflammatory cytokine, IL-10, was the principal driver of human macrophage PDL1 expression in response to HIV infection. ${ }^{2}$ The aim of this study was to investigate how human lung macrophages regulate their PDL1 expression in response to influenza infection.

Methods Alveolar macrophages washed from resected human lung tissue and purified by plate adherence or human positivelyisolated CD14+ monocyte-derived macrophages (MDMs) were cultured with H3N2 X31 influenza virus or a UV-irradiated aliquot of virus (UVX31) for $2 \mathrm{~h}$, after which the cultures were washed and media replaced and incubation continued for a further $22 \mathrm{~h}$. Virally infected cells and expression of cell surface markers were identified using flow cytometry. Gene expression was measured using RT-PCR.

Results No increase in MDM infection was seen using the UVX31 but incubation with $\mathrm{X} 31$ resulted in an average infection rate of 9.1\%. Infection with X31 significantly increased cell surface expression of HLA-DR and PDL1 ( $p<0.05)$, but not of PDL2 by MDMs as 